\title{
Distribución, superficie y accesibilidad de las áreas verdes en Santiago de Chile
}

Sonia Reyes Päcke Instituto de Estudios Urbanos y Territoriales. Pontificia Universidad Católica de Chile.

Isabel Margarita Figueroa Aldunce Dirección de Planeamiento, Ministerio de Obras Públicas. Chile.

RESUMEN | Las áreas verdes urbanas (AVU) proveen servicios sociales y ecológicos, en función de su distribución, superficie y accesibilidad. Se evalúan dichos atributos para las AVU de Santiago mediante métricas de paisaje. Se muestra que el 91\% de las AVU tiene tamaño menor a $5.000 \mathrm{~m}^{2}$. Las comunas de bajos ingresos y mayor población presentan un mayor número de áreas verdes (NP), pero de menor tamaño $\left(\leq 1.000 \mathrm{~m}^{2}\right)$. Las cuatro comunas de más altos ingresos concentran el 32,2\% de la superficie total de áreas verdes, mientras que las cuatro comunas más pobres sólo tienen el 4,1\%. El indicador de accesibilidad propuesto (IAc) muestra que en La Pintana (bajos ingresos) el 19,6\% de la población tiene acceso a un área verde de al menos $5.000 \mathrm{~m}^{2}$ cerca de su vivienda (300 m); en San Miguel (ingresos medios) el $45,3 \%$ y en Vitacura (altos ingresos) el 74,1\% de la población. La normativa urbana chilena favorece la proliferación de AVU de pequeño tamaño.

PALABRAS CLAVE | Indicadores ambientales, planificación urbana, sustentabilidad urbana, desigualdad social.

ABSTRACT | Green spaces provide social and ecological functions within urban environments. The magnitude of these depends on the size, distribution and accessibility of such spaces. These attributes of the green areas in Santiago de Chile are evaluated using landscape metrics. The results show that $91 \%$ of green spaces are smaller than 5,000 m2. Municipalities with higher population and lower income levels had a greater number of green spaces, but these are smaller than $1,000 \mathrm{~m} 2$. The four municipalities with the highest income levels have 32.2\% of the total green space surface, while the four municipalities with the lowest income levels barely exceed $4.0 \%$. The proposed accessibility indicator (IAc) reveals that in the La Pintana municipality (low income level), $19.6 \%$ of the population has access to a green space of at least 5,000 within $300 m$ from their home, while this figure is $45.3 \%$ for San Miguel (medium income level) and $74.1 \%$ in Vitacura (high income level). Chilean urban legislation tends to promote the creation of smaller green spaces.

KEY WORDS | Environmental indicators, urban planning, urban sustainability, social inequality. 


\section{Introducción}

Las áreas verdes urbanas son elementos fundamentales para mejorar el bienestar de la población urbana, especialmente en grandes ciudades. Sin embargo son escasas en las grandes ciudades de América Latina, producto de la historia de urbanización precaria y explosiva de la segunda mitad del siglo XX. El Área Metropolitana de Santiago (AMS) comparte esta realidad y, aunque en las últimas décadas se han realizado importantes inversiones en construcción y recuperación de áreas verdes, persisten grandes diferencias de dotación al interior de la ciudad (De la Maza et al., 2002; Escobedo et al., 2006; Figueroa, 2009).

Debido a las altas tasas de urbanización, las áreas verdes son cada vez más importantes como espacios de interacción entre las personas y también con la naturaleza, generando oportunidades para una mayor interacción social. Asimismo, la frecuencia de interacción social en las áreas verdes es un factor que refuerza el apego a la comunidad y entre los residentes e incluso tiene positivos efectos en la salud de las personas (Maas et al., 2009).

El tamaño y distribución de plazas, parques y áreas verdes tiene implicancias ecológicas y sociales. Desde el punto de vista ecológico, el mayor tamaño de estas áreas permite una mayor diversidad y riqueza de especies vegetales, lo cual va acompañado también de una mayor diversidad de fauna (Kühn et al., 2004; Knapp et al., 2008). Si además se favorece la plantación de árboles y arbustos nativos, aumenta la presencia de aves nativas (Díaz y Armesto, 2003; White et al., 2005) contribuyendo a la conservación de la biodiversidad local.

Las áreas verdes de gran tamaño contribuyen más efectivamente a la regulación de las inundaciones ocasionadas por la acumulación de aguas lluvias, ya que mantienen una alta permeabilidad del suelo y su capacidad de infiltración (Sorensen et al., 1998). La cobertura vegetacional en los parques situados en las laderas de los cerros contribuye a la retención del suelo y disminuye el riesgo de procesos de remoción en masa (Romero y Vásquez, 2005). La contribución de la vegetación a la regulación de la temperatura urbana ha sido demostrada en diversas ciudades (Sorensen et al., 1998; Jenerette et al., 2007), así como la capacidad de capturar partículas y renovar el aire (Hough, 1998; Hernández, 2007).

Desde el punto de vista social, un mayor tamaño de las áreas verdes -y de los espacios públicos en general- permite la realización de diversas actividades y con ello facilita la presencia simultánea de distintos grupos, por ejemplo niños, adultos y jóvenes. Los distintos grupos de edad tienen diversos requerimientos y utilizan de distinta forma los espacios públicos (Tahvanainen et al., 2001; Chiesura, 2004; Mäkinen y Tyrväinen, 2008; Sugiyama y Ward-Thompson, 2008), razón por la cual la provisión de zonas diferenciadas es un factor que favorece la interacción entre ellos y la integración social en la escala del barrio o la comuna.

Existe una relación directa entre el tamaño y área de servicio de las áreas verdes. Las áreas verdes de mayor tamaño pueden encontrarse en menor número y más alejadas, en cambio las plazas o pequeñas áreas que se encuentran al interior de los ba- 
rrios debieran encontrarse a una distancia tal que se pueda acceder en un tiempo no superior a 10 o 15 minutos de caminata (Coles y Bussey, 2000; Handley et al., 2003).

El indicador utilizado en Chile para evaluar la dotación de áreas verdes urbanas es la superficie total de áreas verdes dividida por el número de habitantes, teniendo como referencia el valor de 9,0 metros cuadrados de área verde por habitante $\left(\mathrm{m}^{2}\right)$ hab.) propuesto por la OMS. Sin embargo, este indicador no entrega información acerca de la accesibilidad de dichas áreas, ni tampoco de su distribución al interior de la ciudad. Los indicadores aplicados en el presente estudio contribuyen a una mejor evaluación de los beneficios sociales y ecológicos de las áreas verdes urbanas en el AMS.

Los estudios realizados en el AMS coinciden al señalar que hay un déficit de áreas verdes, comparado con el estándar de $9,0 \mathrm{~m}^{2} /$ hab. También hay acuerdo respecto a las profundas desigualdades existentes entre las 34 comunas que constituyen el AMS. De acuerdo con un catastro realizado por la Comisión Nacional de Medio Ambiente, en el año 2003 el promedio metropolitano era de $3,2 \mathrm{~m}^{2} / \mathrm{hab}$., pero las comunas más pobres registraban valores muy inferiores, entre 2,9 y $0,4 \mathrm{~m}^{2} /$ hab., mientras que las comunas de más altos ingresos tenían entre 6,7 y 18,8 m²/hab. (Nilo, 2003). En el año 2009 el promedio era 3,9 $\mathrm{m}^{2} / \mathrm{hab}$., con valores extremos de $1,1 \mathrm{~m}^{2} / \mathrm{h}$ ab. en Quinta Normal, y 12,6 $\mathrm{m}^{2} /$ hab. en Santiago (www.observatoriourbano.cl). Esta última medición sólo consideró las áreas verdes con mantenimiento municipal.

Si bien el diagnóstico respecto al déficit de áreas verdes en Santiago de Chile es compartido, hay aspectos relevantes todavía poco estudiados, tales como la distribución espacial, tamaño, dispersión y grado de fragmentación. Asimismo, los catastros realizados por instituciones públicas se refieren solamente a las áreas verdes de propiedad fiscal y, en algunos casos, sólo a aquellas que cuentan con mantenimiento, por tanto no son exhaustivos. El objetivo de este trabajo ha sido conocer la superficie total de áreas verdes en Santiago de Chile y algunos atributos relevantes, tales como distribución, accesibilidad, tamaño y dispersión, con el fin de obtener un diagnóstico más completo acerca de la situación actual y contribuir al diseño de políticas urbanas más eficaces.

\section{Metodología}

\section{Área de Estudio}

El AMS está localizado en los 3327’ de latitud Sur, $70^{\circ} 42^{\prime}$ longitud Oeste a los pies de la Cordillera de los Andes, entre los 700 y 1.000 msnm. Es la capital administrativa, política y económica del país, con una población de 5,79 millones de habitantes (INE, 2005) y una densidad promedio de 93,3 hab./hectárea. Se divide en 34 comunas, pero no constituye una unidad político-administrativa, ni cuenta con un gobierno metropolitano. Se caracteriza por un clima mediterráneo semiárido, con precipitaciones invernales y estación seca prolongada. La precipitación promedio anual es de $350 \mathrm{~mm}$., con marcadas oscilaciones interanuales. Estas características climáticas son extraordinariamente relevantes para el diseño y mantención de áreas verdes y parques urbanos. 


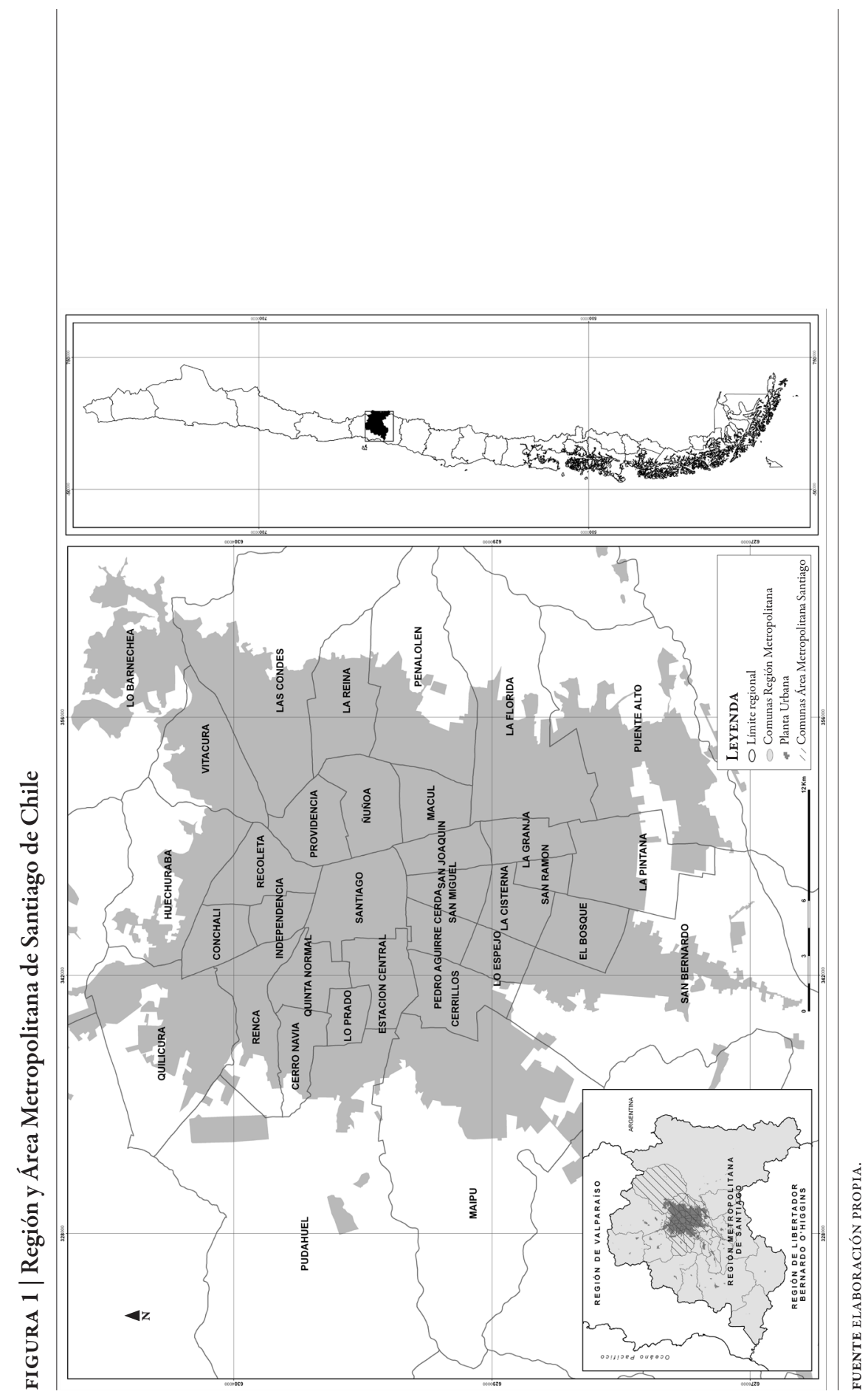




\section{Mapeo y Análisis de las áreas verdes}

La identificación y análisis de las áreas verdes fueron realizados con ArcGis 9.2. Todas las áreas verdes fueron digitalizadas desde fotografías aéreas georreferenciadas, escala 1:20.000, del Servicio Aerofotogramétrico (SAF) dependiente de la Fuerza Aérea de Chile. Las fotografías fueron tomadas en febrero de 2006, en el verano del Hemisferio Sur.

Para caracterizar y describir las áreas verdes del AMS en términos de su composición y distribución espacial, se aplicaron métricas de paisaje: superficie total de áreas verdes por comuna (CA), porcentaje de la superficie total de áreas verdes del AMS (PLAND), número de áreas verdes (NP), índice del fragmento más grande (LPI) - que entrega la participación del área verde más grande respecto de la superficie total de áreas verdes de la comuna- y el Índice de Cohesión (IC), que entrega el grado de conectividad estructural o física de las áreas verdes. Para complementar este último indicador se aplicó el índice del vecino más cercano (NNR). Los indicadores fueron obtenidos con ArcGis 9.2 y Fragstats 3.3. Los datos fueron consolidados a escala comunal, porque en Chile la mantención de las áreas verdes corresponde a dicho nivel administrativo. Se determinó el promedio, desviación estándar, media y mediana para cada indicador (NP, CA, PLAND, LPI, IC y NNR). Esto fue complementado con análisis de correlación para identificar posibles relaciones entre los indicadores.

\section{Análisis de accesibilidad}

La población por comuna se obtuvo del Censo de Población y Vivienda del año 2002, elaborado por el Instituto Nacional de Estadísticas (INE). Para clasificar la población según sus ingresos se utilizó la clasificación de $\mathrm{ADIMARK}^{1}$ que divide a la población en cinco grupos de ingreso: $\mathrm{ABC} 1, \mathrm{C} 2, \mathrm{C} 3, \mathrm{D}$ y E. El grupo $\mathrm{ABC} 1$ es el de más altos ingresos y los grupos D-E son los más pobres. Esta clasificación considera dos variables medidas por hogar en base al Censo 2002 del Instituto Nacional de Estadísticas: el nivel de educación del jefe de hogar y la tenencia de bienes. La georreferenciación a nivel de manzana censal fue provista por el Observatorio de Ciudades UC (www.ocuc. cl), cuyas fuentes de información fueron la población por manzana del Censo 2002, del Instituto Nacional de Estadísticas, manzanas del proyecto "Sistema de Indicadores Comunales" (SIDICO) de la Secretaría Regional Ministerial de Vivienda y Urbanismo (SEREMI MINVU), permisos de edificación años 2002 y 2005 y datos de la Encuesta Nacional de Estratificación Social (CASEN) del año 2006.

Para relacionar la distribución de los grupos socioeconómicos con la distribución de las áreas verdes, la información se expresa a nivel de manzana, incluyendo número de habitantes, número de hogares y nivel de ingresos de los hogares. Mediante ArcGis 9.2 se superpusieron los diferentes mapas, en la misma proyección y escala.

La accesibilidad de las áreas verdes se midió mediante el módulo Network Analyst de ArcGis 9.2, a 300 metros lineales, medidos desde el centroide de cada manzana al centroide del área verde más cercana. La distancia fue definida en base al estándar de accesibilidad de áreas verdes propuesto por English Nature y por la Agencia de Medio

Disponible en http://www.adimark.cl/medios/estudios/Mapa_Socioeconomico_de_Chile.pdf 
Ambiente de la Unión Europea. English Nature establece que "las personas no deben vivir a una distancia superior a 300 metros de un área verde natural, de al menos 2 hectáreas de superficie" (Handley et al., 2003, p. 15). La Agencia de Medio Ambiente de la Unión Europea señala que las personas deben vivir como máximo a $900 \mathrm{~m}$ de un área verde (Barbosa et al., 2007). Sin embargo, en Santiago la mayoría de las áreas verdes tiene un tamaño menor al definido por dichas agencias. Por lo tanto, se mantuvo la distancia de 300 metros propuesta por English Nature, pero se disminuyó el tamaño mínimo del área verde a considerar en esta evaluación. El estándar aplicado fue el siguiente: \% de la población comunal que cuenta con un área verde de superficie mayor o igual a $5.000 \mathrm{~m}^{2}$ a una distancia no superior a 300 metros de su vivienda.

Sobre la base de la distribución de los grupos socioeconómicos del AMS se escogieron tres comunas, para analizar cómo se comporta el indicador de accesibilidad de acuerdo al nivel socioeconómico predominante. Las comunas seleccionadas fueron La Pintana (bajos ingresos), San Miguel (ingresos medios) y Vitacura (altos ingresos).

\section{Resultados}

\section{Superficie, número y tamaño de las áreas verdes en el Área Metropolitana de Santiago (AMS)}

Las áreas verdes del AMS totalizan 3.825 ha de superficie. Aquellas de tamaño igual o superior a una hectárea constituyen sólo el $3 \%$ del total $(\mathrm{n}=358)$ pero representan el 62\% (2.387 hectáreas) de la superficie total de áreas verdes (Figueroa, 2009). Esto significa que más de la mitad de la superficie de áreas verdes en Santiago se concentra en sólo el 3\% del número total de áreas verdes, con tamaños mayores a $10.000 \mathrm{~m}^{2}$. Todos los resultados se entregan en los Anexos de este artículo.

CUADRO 1 | Superficie, número y porcentaje del total AMS, por grupos de tamaño

\begin{tabular}{rcc}
\hline $\begin{array}{r}\text { RANGOS DE TAMAÑ DE LAS } \\
\text { ÁREA VERDES }\end{array}$ & $\begin{array}{c}\text { NÚMERO DE ÁREAS VERDES } \\
\text { POR RANGO DE TAMAÑo }\end{array}$ & $\begin{array}{c}\text { PORCENTAJE DEL TOTAL DE } \\
\text { ÁREAS VERDES AMS }\end{array}$ \\
\hline $500 \mathrm{M}^{2}$ & 3.813 & $32,90 \%$ \\
$1.000-5.000 \mathrm{M}^{2}$ & 2.912 & $25,10 \%$ \\
$\geq 5.000 \mathrm{M} 2$ & 4.072 & $35,10 \%$ \\
TOTAL & 809 & $7,00 \%$ \\
\hline
\end{tabular}

FUENTE BASADO EN FIGUEROA, 2009.

Las comunas con menor superficie de áreas verdes, de acuerdo al índice de CA son: Independencia (17,4 ha), San Miguel (21,4 ha), Quinta Normal (25,3 ha), Lo Espejo (30,9 ha) y El Bosque (31,0 ha) (Figura 3). Todas tienen una mayor concentración de estratos socioeconómicos D y E, a excepción de San Miguel que tiene mayor presencia de estratos medios. 
En el extremo opuesto, con las mayores superficies de áreas verdes se encuentran Vitacura (458,1 ha), Recoleta (293,1 ha), Maipú (238,6 ha), Renca (238,3 ha) y Las Condes (221,8 ha). (Figura 2). Vitacura y Las Condes son comunas de altos ingresos, con mayor presencia de grupos socioeconómicos $\mathrm{ABC} 1$ y presentan las mayores superficies de áreas verdes consolidadas de la ciudad. Recoleta y Renca presentan un predominio de grupos socioeconómicos D, E y en menor medida C3, y el alto valor de CA se debe a la presencia de las dos áreas verdes más extensas del AMS: Parque Metropolitano y Cerros de Renca. El primero está muy bien consolidado y gran parte de su extensión está en las comunas de Recoleta y Providencia. El segundo, en cambio, que está íntegramente en la comuna de Renca, sólo tiene 2 hectáreas completamente habilitadas y el resto de la superficie presenta alguna cobertura vegetal, producto de diversos planes parciales de reforestación. Por último, Maipú es una de las comunas más populosas del AMS, con predominio de grupos socioeconómicos medios ( 2 y C3) y la superficie de áreas verdes está subdividida mayoritariamente en polígonos de tamaño inferior a $1.000 \mathrm{~m}^{2}$.

FIGURA 2 | Superficie de áreas verdes por comuna, AMS

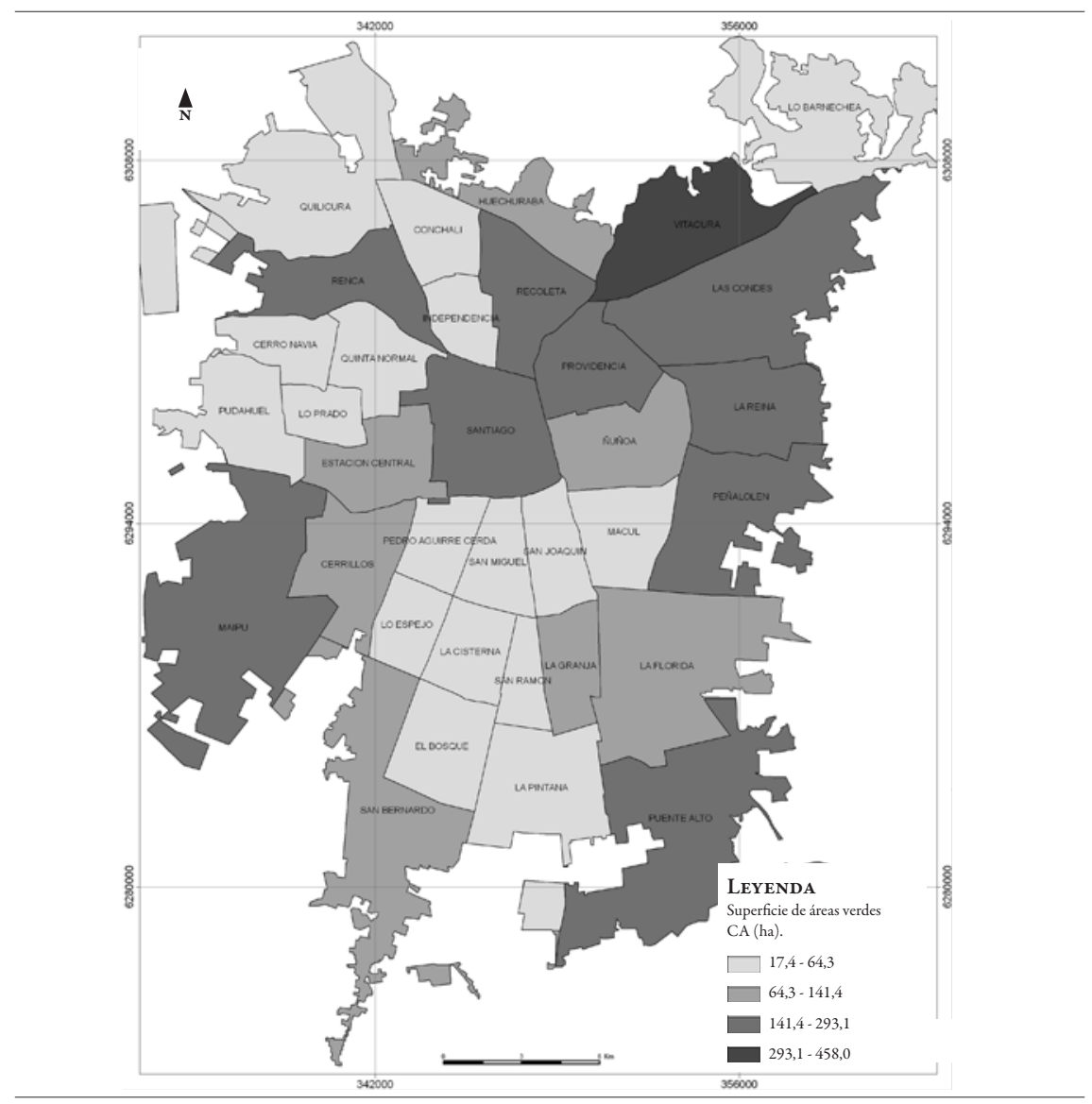

FUENTE FIGUEROA, 2009. 
La densidad de áreas verdes (superficie de área verde por manzana) muestra la concentración de éstas en algunos puntos del AMS, asociados a la presencia de grandes parques: Parque Metropolitano, Parque Mahuida, Parque Padre Alberto Hurtado y Parque Cousiño Macul, entre otros. En el resto de la ciudad la densidad de áreas verdes es baja, en particular en la periferia Sur y Poniente de la ciudad. (Figura 3).

FIGURA 3 | Densidad de áreas verdes por comuna, AMS

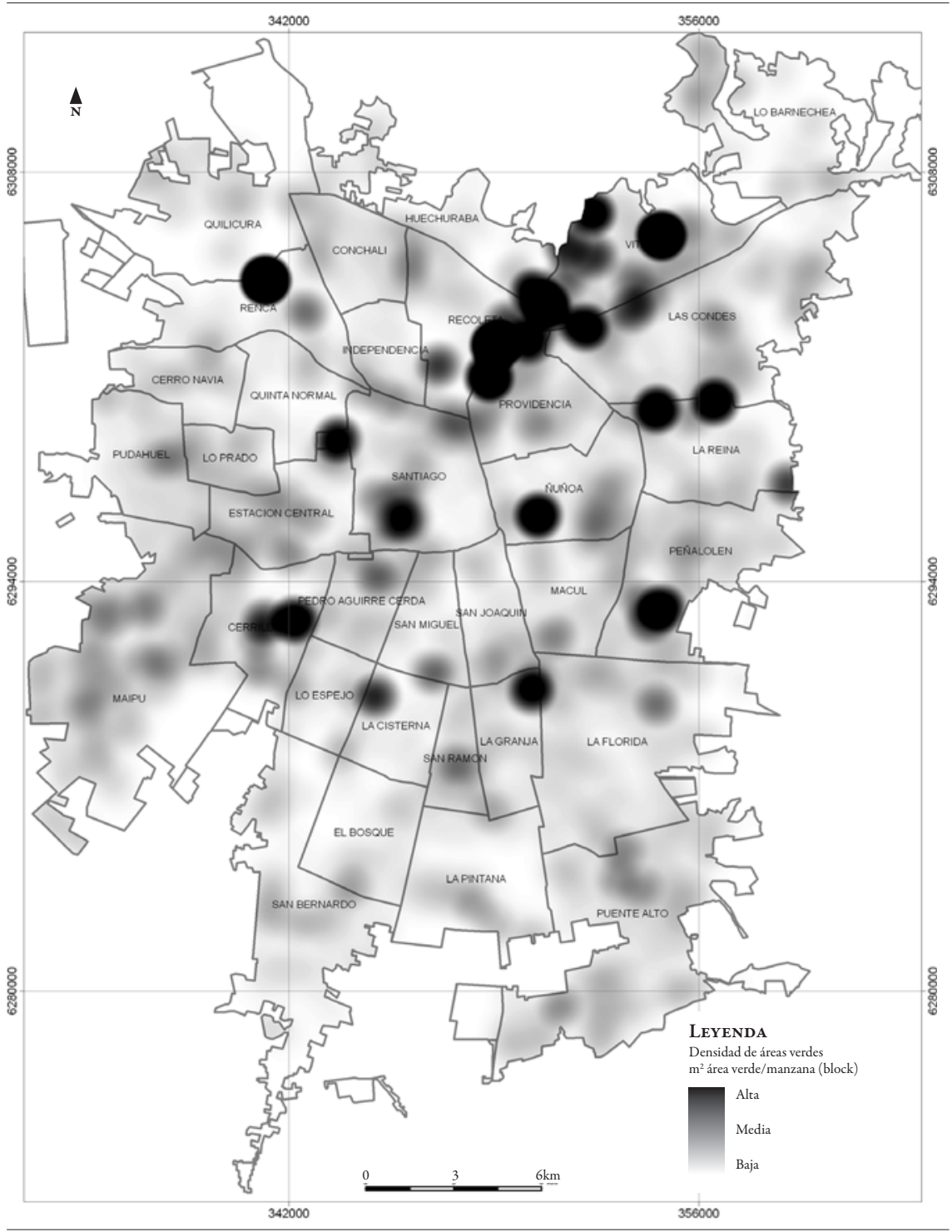

FUENTE ELABORACIÓN PROPIA, BASADO EN FIGUEROA, 2009. 
La situación del número de áreas verdes (NP) es muy distinta, puesto que en este caso se encuentra una alta correlación con el número de habitantes $\left(\mathrm{R}^{2}=0.9\right)$ y no así con su nivel socioeconómico (Figueroa, 2009) (Gráfico 1). Las comunas de Maipú y Puente Alto presentan altos valores de NP pero no cuentan con ningún parque importante en su territorio. Ambas corresponden a las comunas más pobladas del país con 492.915 y 468.390 habitantes en el año 2002 respectivamente (SINIM, 2009), y presentaron altas tasas de crecimiento en el último período intercensal: Maipú incrementó su población en 82,6\% y Puente Alto en 93,5\% (cálculo basado en datos censales). En ambos casos la expansión urbana se aceleró desde el año 1982, debido a la construcción masiva de viviendas económicas.

Las comunas que tienen los mayores valores de NP se localizan en la periferia del AMS: en el sector oriente (Peñalolén, La Florida y Las Condes), Sur (Puente Alto) y Poniente (Maipú). Mientras que los menores valores de NP se presentan en comunas pericentrales (tales como La Cisterna, Quinta Normal, San Miguel e Independencia), a excepción de Renca que se localiza en la periferia Norte del AMS.

GRÁFICO 1 | Correlación entre el Número de áreas verdes (NP) y la población comunal

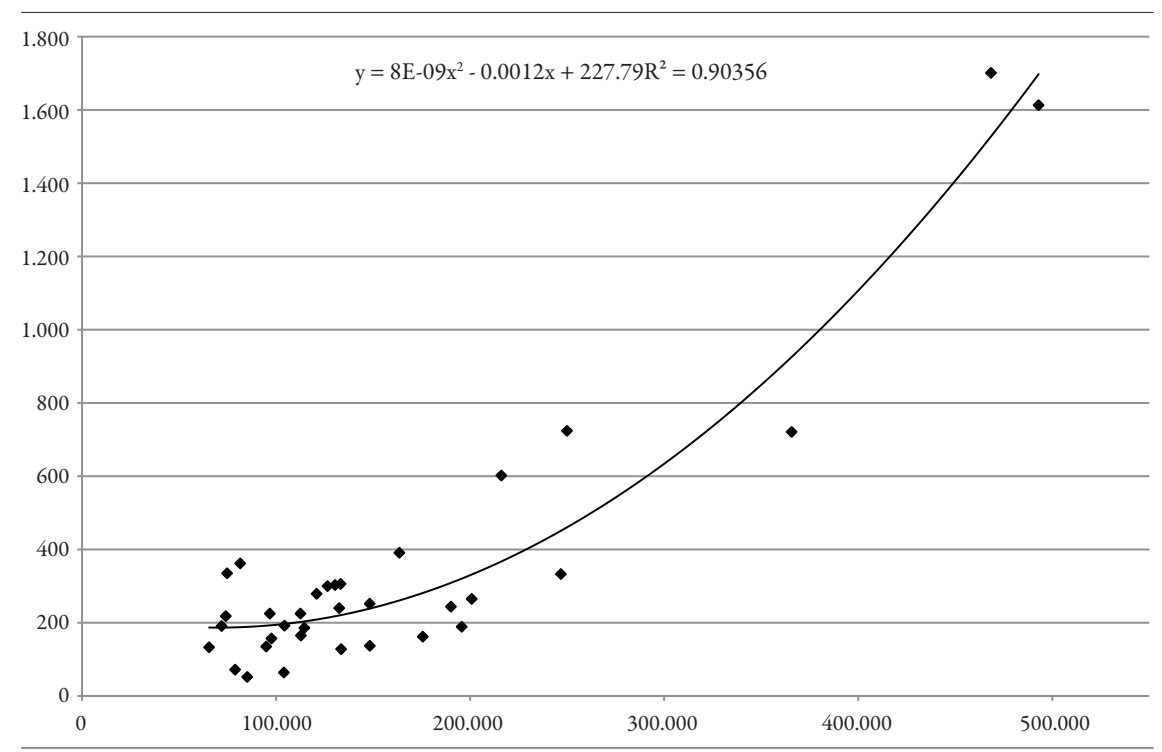

FUENTE FIGUEROA, 2009.

Distribución de las áreas verdes y grandes parques (resultados PLAND y LPI)

PLAND expresa la participación de cada comuna en la superficie total de áreas verdes del AMS. Dado que cada comuna tiene distinta población y distinta superficie, este indicador tiene un valor comparativo, permitiendo detectar las diferencias entre comunas. Pero debe ser complementado con otros indicadores para establecer conclusiones o recomendaciones válidas. Con valores de PLAND inferiores 
al 2\% se encuentran 17 comunas; con valores comprendidos en el rango 2 -4\% se encuentran 7 comunas, y sobre el $4 \%$ se encuentran 10 comunas, destacando entre éstas Recoleta con PLAND=7,66\% y Vitacura con PLAND $=11,98 \%$ (Figueroa, 2009) (ver Gráfico 2).

GRÁfICO 2 | Distribución de valores de PLAND por comuna AMS

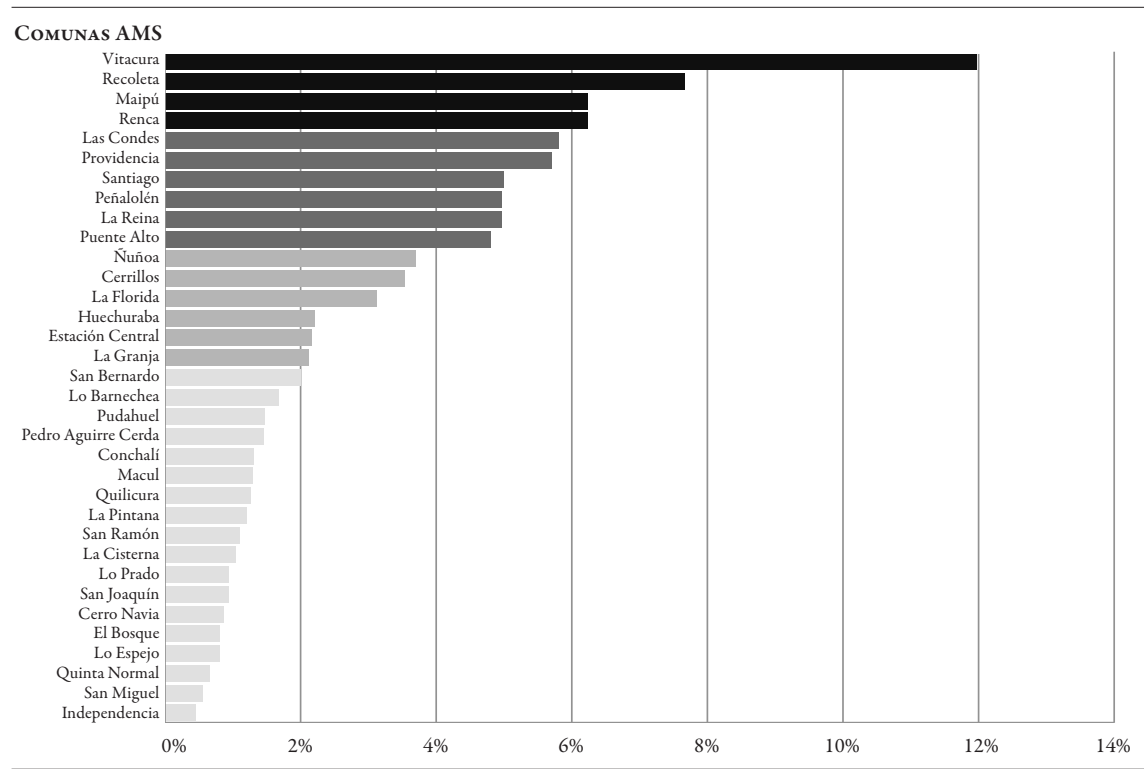

FUENTE BASADO EN FIGUEROA, 2009.

Tanto por la importancia social como ambiental, se evaluaron la presencia de grandes parques y la participación de ellos en la superficie total de áreas verdes comunales, mediante el índice LPI (Largest Patch Index). Éste indica la participación del área verde de mayor extensión en la superficie total de áreas verdes de cada comuna. Las comunas que presentan los valores más bajos de LPI no poseen áreas verdes de gran extensión. Así se tiene que 19 comunas presentan valores inferiores al 20\%, es decir, no contienen ningún área verde que represente hasta el $20 \%$ de la superficie total de áreas verdes de la comuna (Gráfico 3). Los valores más altos se registran en Renca (LPI=84,9) correspondiente al Cerro Renca; Recoleta $(\mathrm{LPI}=77,4)$ y Providencia (LPI=66,7) debido a la presencia del Parque Metropolitano. La comuna de La Reina, a pesar de contar con el Parque Mahuida en su territorio, presenta un valor de LPI medio (LPI=33,8) debido a la gran superficie de áreas verdes de menor tamaño dispersas en la comuna y a la presencia del Parque Padre Hurtado, lo cual hace que la participación del Parque Mahuida en el total de áreas verdes de la comuna sea ligeramente menor al 34\%. 
GRÁfICO 3 | Distribución de valores de LPI por comuna AMS

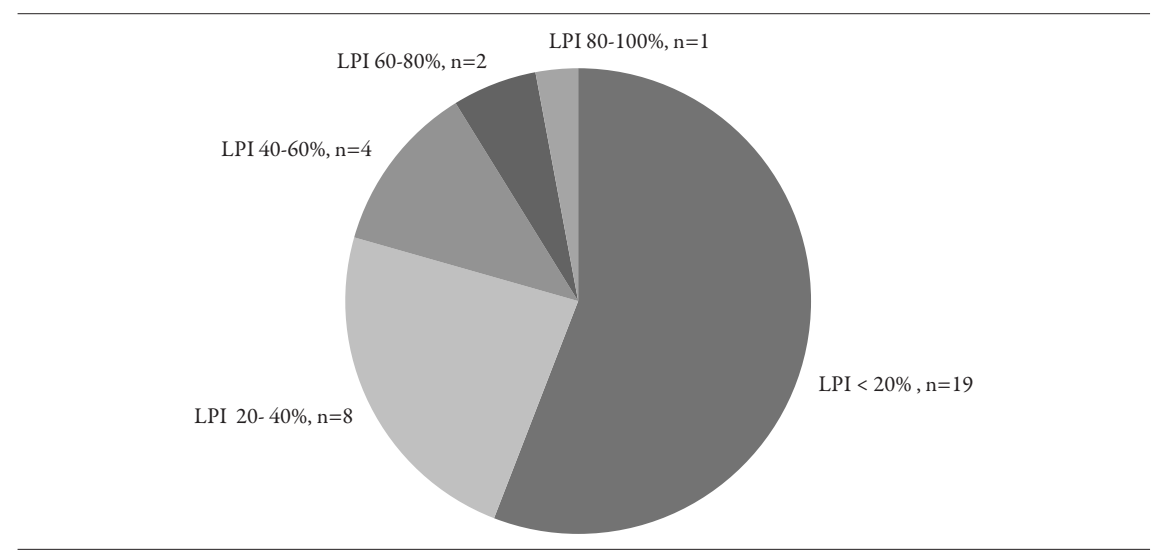

FUENTE BASADO EN FIGUEROA, 2009.

\section{Conectividad estructural de las áreas verdes (IC, NNR)}

Respecto del índice de cohesión (IC), todas las comunas tienen valores superiores a 90, lo cual significa que hay un patrón de distribución concentrado, a pesar de las diferencias en número o superficie de áreas verdes. La escasa dispersión de los resultados del índice de cohesión es producto de que la disposición espacial de las áreas verdes responde al diseño de la ciudad y su trama urbana, lo cual establece un patrón de distribución regular de los elementos de la urbanización, a diferencia de lo que sucede en los espacios rurales en donde la disposición de los elementos suele ser más irregular. Ello es consistente con lo señalado por Saura y Martínez Millán (2001), en el sentido de que la variabilidad de este índice es muy baja cuando el patrón de paisaje ocupa gran parte del área estudiada, porque el IC es menos sensible a los cambios en la configuración espacial del paisaje cuando la abundancia de la clase (áreas verdes) es alta (Saura y Martínez Millán, 2001) (Neel et al., 2004). Las condiciones señaladas se cumplen en los espacios urbanos: alto grado de fragmentación de las áreas verdes y configuración espacial bastante regular. En este análisis resultan más relevantes las variaciones del indicador entre comunas que el valor absoluto obtenido, ya que permite diferenciar entre aquellas que tienen mayor o menor conectividad.

Se identificó una estrecha relación entre el índice de cohesión (IC) y el LPI $\left(\mathrm{R}^{2}=0.7\right)$. Las comunas que superan el $50 \%$ del valor de LPI tienen altos valores de conectividad y bajo el 40\% de LPI se aprecia una mayor dispersión de los datos. De acuerdo con estos resultados, la presencia de un área verde de tamaño mayor, que supere el 50\% de la superficie total de las áreas verdes contribuye a mejorar la conectividad entre ellas.

Los resultados obtenidos para el Índice del vecino más cercano (NNR) varían desde 0,45 en el caso de Huechuraba, hasta 0,9 en Macul. Pese a la diferencia entre el valor mínimo y máximo, la distribución de los valores es homogénea, ya que el promedio es igual a la mediana $(0,71)$ y tiene una pequeña desviación estándar $(\mathrm{DS}=0,1)$. Las clases fueron definidas por el método reiterativo y el histograma 
de frecuencia muestra una distribución normal. Este indicador confirma que las áreas verdes en todas las comunas presentan un patrón concentrado. A escala de ciudad también se aprecia una distribución agrupada, ya que el valor del NNR para el AMS es de 0,59 con un 1\% de probabilidad que este patrón sea al azar.

\section{Accesibilidad de áreas verdes $\geq 5.000 \mathrm{~m}^{2}$ a 300 metros}

El Índice de Accesibilidad (IAc) fue aplicado en tres comunas del AMS: La Pintana, San Miguel y Vitacura, con el fin de identificar potenciales diferencias entre comunas con distinto perfil socioeconómico. Las diferencias se pueden apreciar en el distinto número de áreas verdes en cada comuna, pero se agudizan al considerar que La Pintana tiene 2,5 veces la población de Vitacura, y que en esta última hay un mayor porcentaje de las áreas verdes en buen estado. Así también, Vitacura contiene más del doble del número de áreas verdes $\geq 5.000 \mathrm{~m}^{2}$ que las otras dos comunas, confirmando que la comuna con mayor nivel de ingresos tiene mejor dotación de áreas verdes.

CUADRO 2 | Número y porcentaje de áreas verdes según rangos de tamaño $\left(\mathbf{m}^{2}\right)$, población comunal y valor de IAc por comuna

\begin{tabular}{|c|c|c|c|c|c|c|}
\hline \multirow{2}{*}{$\begin{array}{l}\text { RANGO DE TAMAÑo DE } \\
\text { LAS ÁREAS VERDES }\left(M^{2}\right)\end{array}$} & \multicolumn{2}{|c|}{ La Pintana } & \multicolumn{2}{|c|}{ San Miguel } & \multicolumn{2}{|c|}{ Vitacura } \\
\hline & $\mathrm{n}$ & $\%$ & $\mathbf{n}$ & $\%$ & $\mathbf{n}$ & $\%$ \\
\hline $4-999,9$ & 126 & 51,6 & 28 & 38,9 & 205 & 56,6 \\
\hline $1.000-4.999,9$ & 108 & 44,3 & 33 & 45,8 & 107 & 29,6 \\
\hline$\geq 5.000$ & 10 & 4,1 & 11 & 15,3 & 50 & 13,8 \\
\hline Total áreas verdes & 244 & 100 & 72 & 100 & 362 & 100 \\
\hline Población comunal & \multicolumn{2}{|c|}{173.462} & \multicolumn{2}{|c|}{76.716} & \multicolumn{2}{|c|}{73.048} \\
\hline $\begin{array}{r}\mathrm{N}^{\circ} \text { habitantes con área } \\
\text { verde } \geq 5.000 \mathrm{~m}^{2} \text { a } 300 \mathrm{~m} . \\
\text { de la vivienda }\end{array}$ & \multicolumn{2}{|c|}{33.929} & \multicolumn{2}{|c|}{34.760} & \multicolumn{2}{|c|}{54.106} \\
\hline $\begin{array}{r}\text { Iac (\% población con área } \\
\text { verde } \geq 5.000 \mathrm{~m} 2 \text { a } 300 \mathrm{~m} . \\
\text { de la vivienda) }\end{array}$ & \multicolumn{2}{|c|}{19,6} & \multicolumn{2}{|c|}{45,3} & \multicolumn{2}{|c|}{74,1} \\
\hline
\end{tabular}

FUENTE ELABORACIÓN PROPIA BASADO EN FIGUEROA, 2009.

En La Pintana sólo el 19,6\% de la población tiene acceso a un área verde $\geq 5.000$ $\mathrm{m}^{2}$ a una distancia máxima de 300 metros de su vivienda (Figura 4). Esta cifra es bastante baja y se debe a que sólo cuenta con diez áreas verdes de ese tamaño, aunque se encuentran en sectores con alta densidad poblacional. El mayor porcentaje de áreas verdes tiene tamaños muy pequeños (menores a $1.000 \mathrm{~m}^{2}$ ), situación que se complejiza al considerar que el 38\% de ellas se encuentra en mal estado (Figueroa, 2009). Sin embargo, el $100 \%$ de las áreas verdes $\geq 5.000 \mathrm{~m}^{2}$ se encuentran en buen estado, lo cual es particularmente importante en una comuna con un $84,4 \%$ de los hogares pertenecientes a los grupos de más bajos ingresos (Universidad de Chile, 2008) y en donde la gran mayoría de las familias habita en viviendas sociales. Las áreas verdes de mayor tamaño corresponden al Parque Mapuhue (6 hectáreas) y el Estadio Municipal (5,7 hectáreas), en las cuales hay campos deportivos y jardines. 
FIgURA 4 | Accesibilidad áreas verdes, comuna de La Pintana, AMS

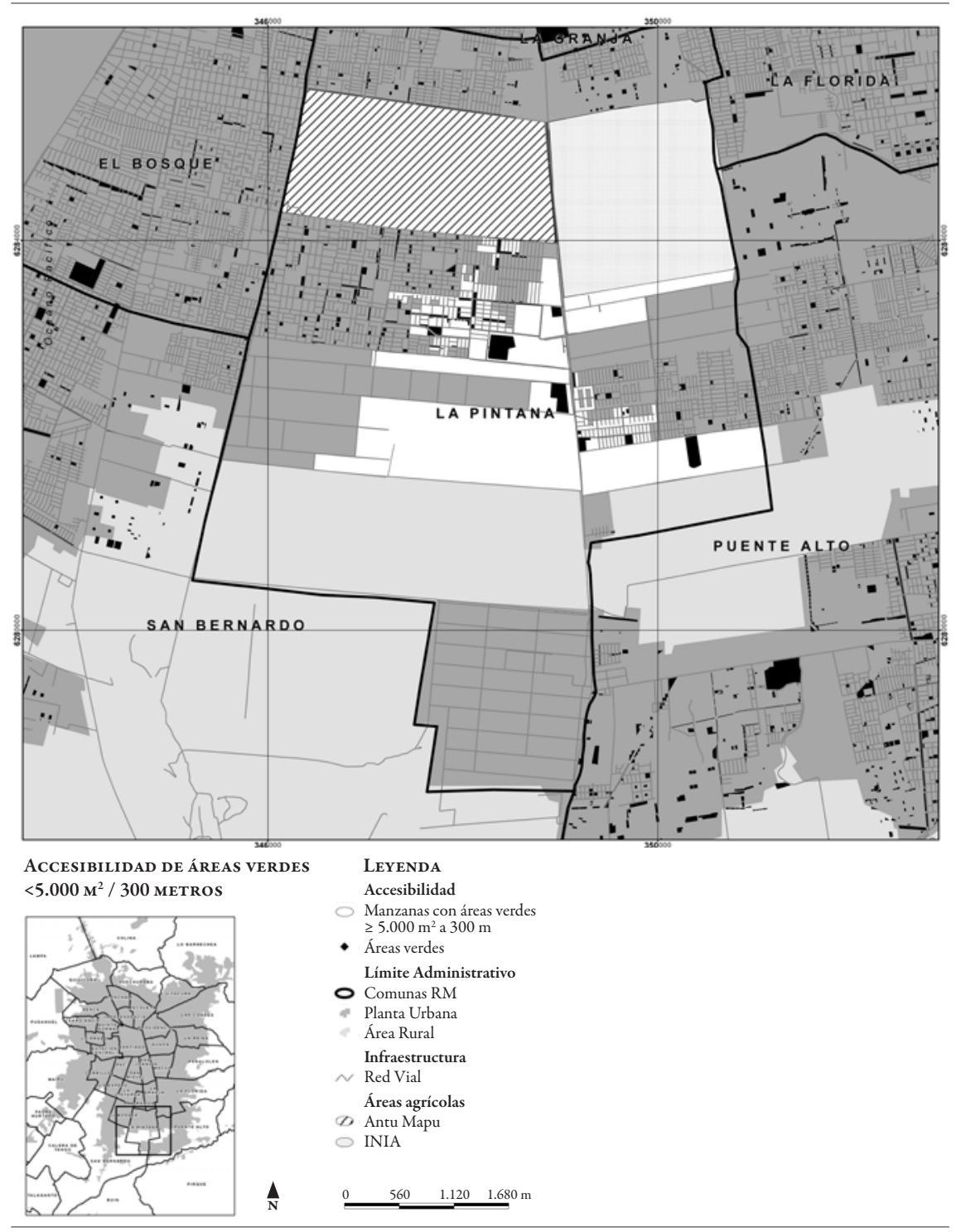

FUENTE ELABORACIÓN PROPIA BASADO EN FIGUEROA, 2009.

En San Miguel el 45,3\% de los habitantes tienen acceso a un área verde $\geq 5.000$ $\mathrm{m}^{2}$ a una distancia máxima de 300 metros de su vivienda, lo que equivale a 34.760 personas (Figura 5). Esta comuna tiene una superficie de 950 hectáreas y se localiza en la zona intermedia del Área Metropolitana de Santiago. Tiene un total de 72 áreas verdes, de las cuales 11 (15,3\%) tienen una superficie mayor a los 5.000 $\mathrm{m}^{2}$ (Figueroa, 2009). Esta distribución de tamaños es más equilibrada que aquella 
observada en La Pintana, porque hay una mayor presencia de áreas de mayor tamaño. Ello se relaciona con el origen de esta comuna, que fue urbanizada en sucesivos períodos, por propietarios individuales, agrupaciones gremiales y de trabajadores y, en menor medida, por diversos programas de vivienda social. La estructura de ingresos de la población también refleja estas características, predominando los estratos medios (C2 y C3).

FIGURA 5 | Accesibilidad áreas verdes, comuna de San Miguel, AMS

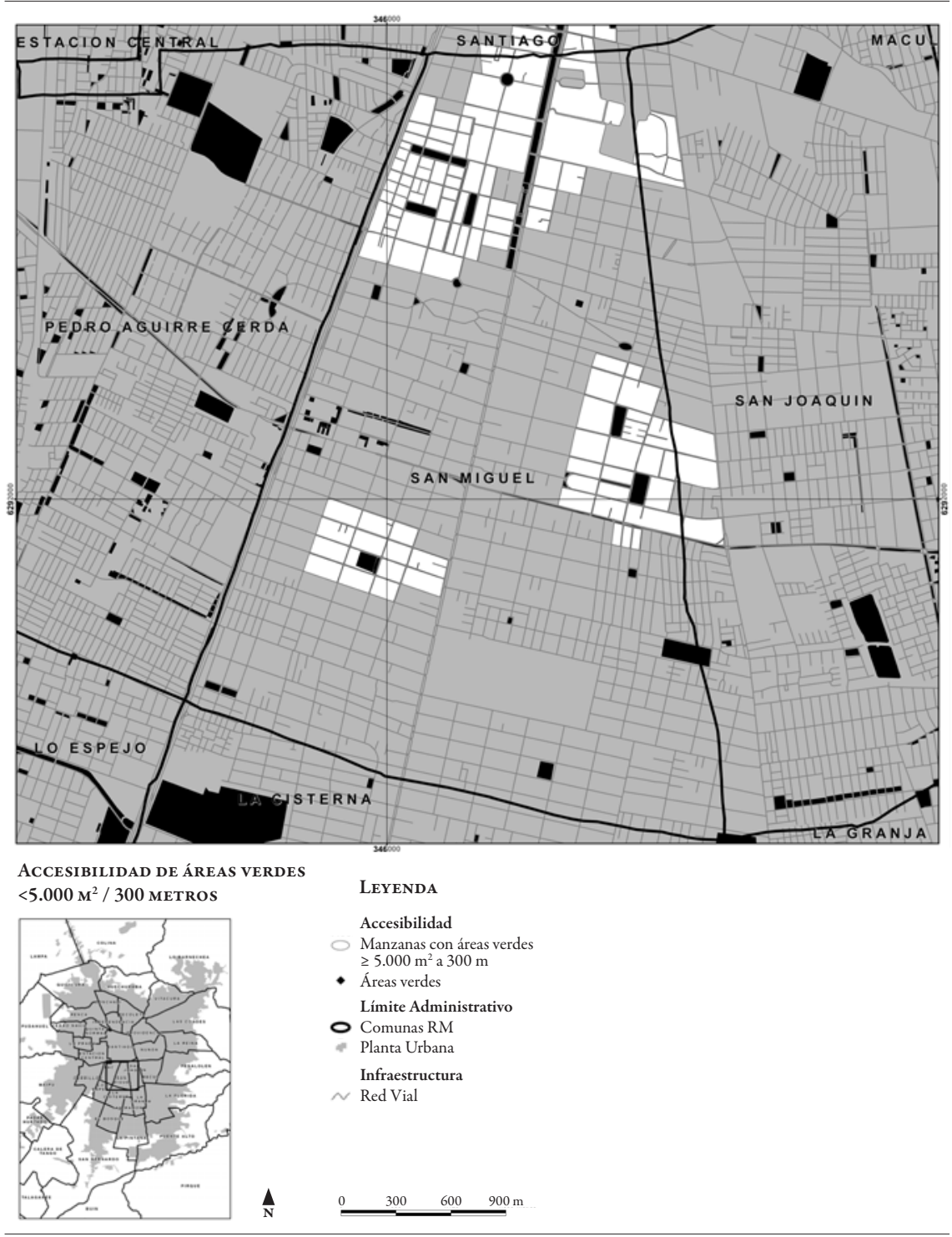

FUENTE ELABORACIÓN PROPIA BASADO EN FIGUEROA, 2009. 
Para este indicador, Vitacura tiene el mayor valor, ya que un $74,1 \%$ de sus habitantes tiene acceso a un área verde $\geq 5.000 \mathrm{~m}^{2}$ a una distancia máxima de 300 metros de su vivienda, lo cual corresponde a 54.106 habitantes (Figura 6). Esta comuna cuenta con 362 áreas verdes, de las cuales 50 (13,8\%) tienen un tamaño superior a los $5.000 \mathrm{~m}^{2}$ (Figueroa, 2009).

FIGURA 6 | Accesibilidad áreas verdes, comuna de Vitacura, AMS

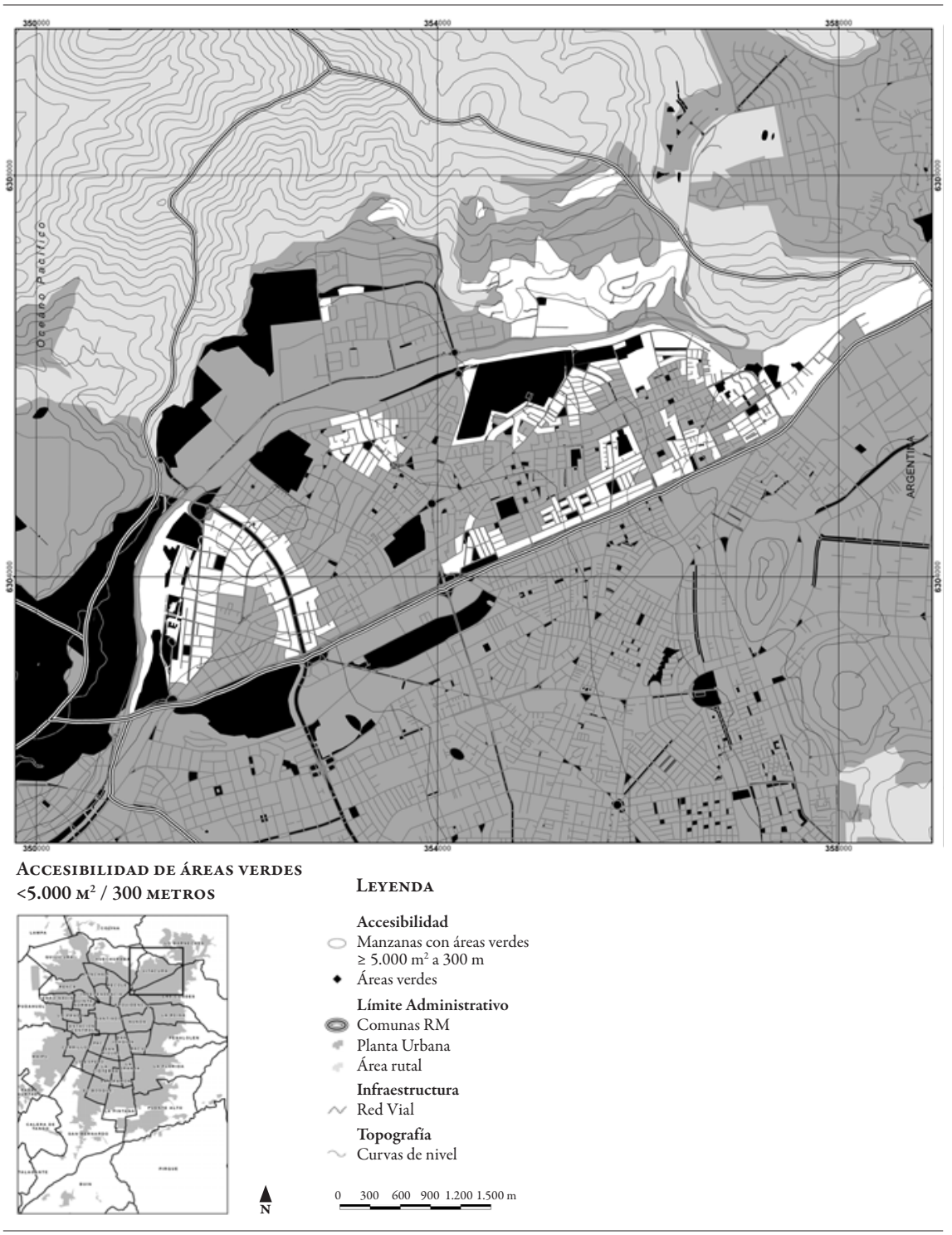

FUENTE ELABORACIÓN PROPIA BASADO EN FIGUEROA, 2009. 
$\mathrm{Al}$ cruzar los datos de accesibilidad con los grupos socioeconómicos, se observa un incremento del acceso a áreas verdes de mayor tamaño en los grupos de mayores ingresos (Gráfico 4). Es decir, los grupos de menores ingresos (D y E) tendrán menos acceso a las áreas verdes de mayor tamaño independientemente de la comuna en la cual estén radicados.

GRÁFICO 4 | Índice de accesibilidad, según grupo socioeconómico de los hogares de comunas analizadas

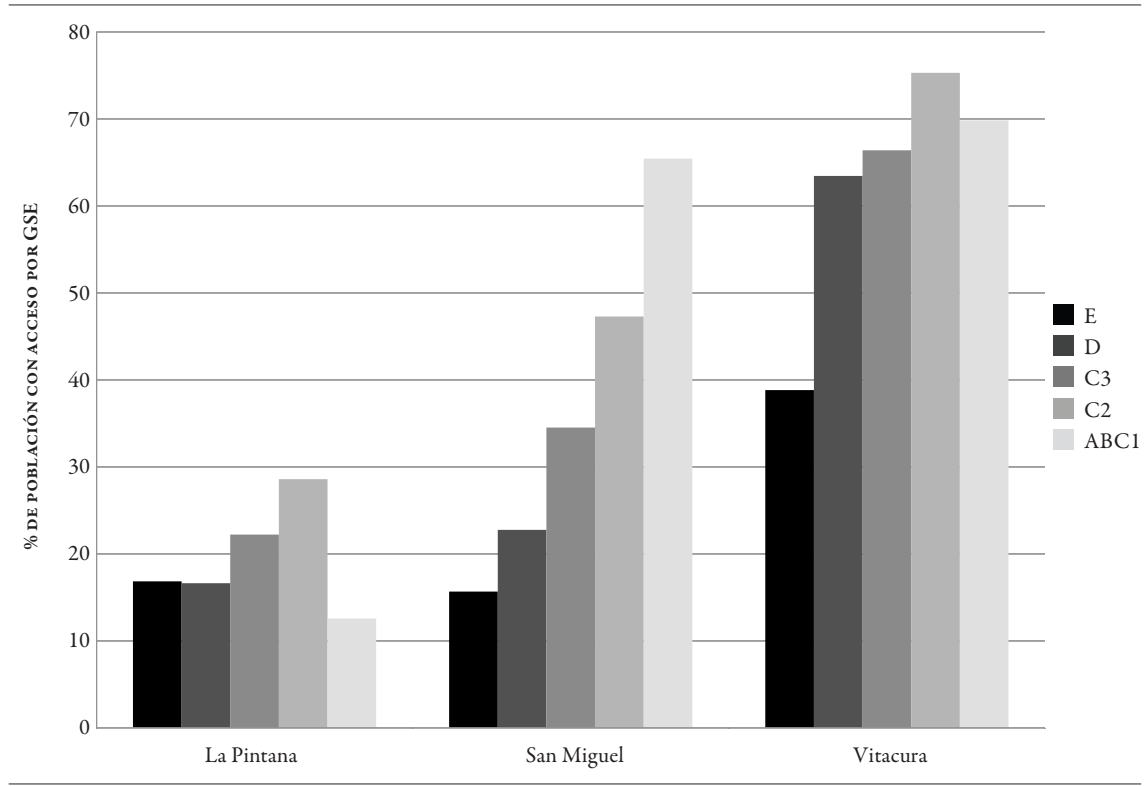

FUENTE ELABORACIÓN PROPIA, EN BASE A FIGUEROA 2009.

\section{Comparación entre indicadores de accesibilidad y superficie área verde/habitante}

El resultado general obtenido en el IAc muestra situaciones diferentes para las tres comunas, en donde la que presenta valores menores es La Pintana, mientras que San Miguel tiene un valor intermedio que se acerca al 50\% de la población y Vitacura es la mejor posicionada con más del $70 \%$ de la población con acceso a 300 metros de su vivienda a un área verde $\leq 5.000 \mathrm{~m}^{2}$.

Por otra parte, al observar el estándar de áreas verdes por habitante propuesto por la OMS ( $9 \mathrm{~m}^{2} / \mathrm{hab}$ como mínimo) se obtiene que La Pintana y San Miguel están bajo dicho estándar (2,4 y $2,7 \mathrm{~m}^{2} /$ habitante, respectivamente), mientras que Vitacura lo supera ampliamente, con $56 \mathrm{~m}^{2} /$ habitante.

Ambos indicadores muestran las diferencias entre las comunas analizadas, las cuales se correlacionan con el nivel socioeconómico de la población. Ambos indicadores son adecuados para comparar la dotación de áreas verdes de comunas o ciudades, pero entregan información en diferentes escalas: el indicador propuesto por la OMS permite conocer de manera genérica y en una primera instancia la situación comunal, mientras que el IAc permite reconocer las diferencias de accesibilidad a una escala más local. 


\section{Conclusiones}

El estudio demuestra que las áreas verdes en Santiago de Chile se caracterizan por una desigual distribución, tanto en términos de superficie total como de tamaño y accesibilidad. Esta desigualdad está altamente correlacionada con el nivel socioeconómico de la población: al comparar las 34 comunas del AMS se encuentra que cuanto mayor es el nivel de ingresos de los hogares, éstos cuentan con mayor superficie total de áreas verdes, las cuales tienen mayor tamaño y mejor accesibilidad. El cuadrante nororiente del AMS (comunas de Vitacura, Las Condes y La Reina) concentra las mayores superficies de áreas verdes de la ciudad, con una extensión hacia el centro, incluyendo las comunas de Recoleta, Providencia y Santiago, situación que se explica por la presencia de los Parques Metropolitano, Forestal, O’Higgins y Quinta Normal, entre los más relevantes. En tanto, la zona Poniente, parte de la zona Norte y el Centro-Sur de la ciudad tienen menor superficie de áreas verdes y éstas son de menor tamaño, lo que coincide con la distribución de los grupos socioeconómicos (Sabatini et al., 2010). En futuros estudios es indispensable evaluar la calidad de las áreas verdes y su relación con el nivel de ingresos de la población.

La utilización de métricas de configuración espacial para el análisis de las áreas verdes urbanas permite evaluar los beneficios sociales y ecológicos de dichas áreas, superando el enfoque basado en la superficie de áreas verdes por habitante. Sin embargo, algunos indicadores son menos sensibles a las características de las ciudades y no resultan tan útiles como en zonas rurales o silvestres. Es el caso del Índice de Cohesión (IC) y el Índice del Vecino más Cercano (NNR), los cuales entregan como resultado un patrón altamente concentrado, con valores similares para todas las comunas del AMS, a pesar de las grandes diferencias en la superficie de áreas verdes. Debido a que la unidad de estudio fue el AMS, se establece el predominio de un patrón de paisaje determinado por la trama urbana.

Una conclusión importante del presente estudio es que los indicadores deben ser utilizados en forma complementaria, puesto que cada uno de ellos permite analizar algún atributo específico de las áreas verdes, pero sólo se puede obtener una visión global analizando el conjunto de ellos. La complejidad del paisaje urbano determina la necesidad de utilizar diferentes indicadores para su descripción.

El índice LPI (Largest Patch Index) permite identificar la existencia de grandes parques, aunque éstos deben estar acompañados de parques y plazas de menor tamaño dispersos en el territorio comunal, para dar cuenta de una óptima distribución de áreas verdes. Asimismo, los resultados indican que la presencia de grandes parques, asociado a un valor de LPI mayor al $50 \%$ contribuye a mejorar la conectividad entre las áreas verdes de la comuna. En el caso del AMS se corrobora la escasez de grandes parques, ya que 24 de las 34 comunas tiene valores de LPI inferiores a $25 \%$ y sólo el 3\% de las áreas verdes tiene una superficie mayor a $10.000 \mathrm{~m}^{2}$.

El alto número de áreas verdes en las comunas periféricas, particularmente en aquellas en donde predominan las urbanizaciones de viviendas sociales, está lejos de ser un indicador positivo, ya que evidencia la extrema fragmentación de estas áreas. 
La normativa urbana vigente favorece la proliferación de pequeñas áreas verdes, ya que no define un tamaño mínimo, sino solamente la obligación de destinar a este uso un porcentaje del terreno que se urbaniza. En este escenario, los urbanizadores destinan a áreas verdes los remanentes de suelo, en los cuales no es posible construir viviendas, cumpliendo sólo con el estándar de superficie exigido. La fórmula establecida para calcular la superficie de áreas verdes que corresponde a zonas residenciales diferencia aquéllas cuya densidad de población es menor a 70 habitantes por hectárea de aquellas que superan dicha densidad (Ministerio de Vivienda y Urbanismo, 2010). Para las primeras, el resultado es la obligación de construir alrededor de $10 \mathrm{~m}^{2}$ de áreas verdes por habitante (Bascuñán et al., 2007). En cambio, en las segundas, el estándar resultante puede ser muy inferior. Bascuñán et al. (2007) han señalado que para las poblaciones de vivienda social, cuyas densidades bordean los 500 habitantes por hectárea, las superficies resultantes oscilan entre 1,1 y $1,5 \mathrm{~m}^{2}$ / habitante, lo cual es consistente con los resultados del presente estudio.

Dado que los beneficios sociales de las áreas verdes dependen de su accesibilidad, se propone un indicador que permita evaluar dicho atributo (IAc=Índice de Accesibilidad). Los estándares de referencia han sido diseñados en Europa (Salvador, 2003; Handley et al., 2003; Barbosa et al., 2007), para ciudades muy distintas al AMS, de manera que se propone un estándar más apropiado a la realidad chilena y latinoamericana: todas las personas deben contar con un área verde de al menos $5.000 \mathrm{~m}^{2}$ a una distancia no mayor a 300 metros de su vivienda.

Los resultados obtenidos reflejan las diferencias entre las comunas estudiadas, pero se evidencia que independientemente de la condición socioeconómica predominante en la comuna, los grupos de más bajos ingresos tienen menor acceso a áreas verdes en su comuna. Ello pone de manifiesto una diferenciación intracomunal, que afecta a los grupos más pobres, quienes, precisamente, requieren de la provisión de servicios urbanos que no pueden adquirir privadamente. Una de las ventajas del indicador de accesibilidad como instrumento de política pública es que permite detectar las carencias al interior de cada comuna (u otra escala territorial) a la vez que permite localizar dichas carencias, porque se construye sobre la base de criterios de distribución espacial.

Sin embargo, la discusión debe centrarse en establecer un tamaño mínimo de un área verde, relacionado con las prestaciones que se esperan o los objetivos que se asignan. Las funciones sociales (encuentro, recreación, contacto con la naturaleza, actividades deportivas) y ecológicas (conservación de biodiversidad, regulación de temperatura urbana, infiltración de aguas lluvia) requieren de una cierta superficie mínima para hacerse efectivas, además de ciertas características de diseño y cobertura vegetacional. Sukopp (1991) establece una superficie mínima de 10 hectáreas, para obtener un aporte ambiental y ecológico significativo (Sukopp y Werner, 1991); English Nature propone un tamaño mínimo de 2 hectáreas (Handley et al., 2003), basado en los requerimientos de los seres humanos; el Plan Verde de Valencia establece un tamaño mínimo de 5 hectáreas para un Parque Urbano (área de servicio en toda la ciudad) y 1 hectárea para Parques distritales (área de servicio co- 
rrespondiente a un distrito o comuna) (Salvador, 2003). Las áreas verdes de menor superficie cumplen algunas funciones ambientales, pero no favorecen la intensidad y diversidad de usos que una comunidad urbana, diversa social y culturalmente, pudiera requerir.

La gran deuda de la ciudad de Santiago con sus habitantes es la provisión de áreas verdes de un tamaño superior a 0,5 hectáreas, así como grandes parques, con tamaño superior a 2 hectáreas. Se necesita una modificación de las regulaciones vigentes, para detener la proliferación de pequeños espacios verdes que sólo tienen un valor estético y cumplen, en su mayoría, funciones complementarias a la vialidad. Este trabajo aporta a la comprensión de un problema complejo, contribuyendo con una visión integral de las áreas verdes urbanas a la formulación de políticas públicas que integran la naturaleza en las ciudades. IEURE

\section{Referencias bibliográficas}

ADIMARK. (s.f.). Mapa socioeconómico de Chile. Recuperado el 19 de julio de 2010, de sitio web: http://www.adimark.cl/medios/estudios/Mapa_Socioeconomico_de_Chile.pdf.

Barbosa, O., Tratalos, J., Armsworth, P., Davies, R., Fueller, R., Pat, J. y otros (2007). Who benefits with access from green space? A case study from Sheffield UK. Landscape and Urban Planning 83, 187-195.

Bascuñán, F., Walker, P. \& Mastrantonio, J. (2007). Modelo de cálculo de áreas verdes en planificación urbana desde la densidad habitacional. Urbano 10, 97-101.

Chiesura, A. (2004). The role of urban parks in a sustainable city. Landscape and Urban Planning 68, 129-138.

Coles, R., \& Bussey, S. (2000). Urban forest landscapes in the UK: progressing the social agenda. Landscape and Urban Planning 52, 181-188.

Comber, A., Brundson, C. \& Green, E. (2008). Using GIS-based network analysis to determine greenspace accesibility for different ethnic and religious groups. Landscape and Urban Planning 86, 103-114.

De la Maza, C., Hernández, J., Bown, H., Rodríguez, M. \& Escobedo, F. (2002). Vegetation diversity in the Santiago de Chile urban ecosystem. Arboricultural Journal 26, 347-357.

Díaz, I. \& Armesto, J. (2003). La conservación de aves silvestres en ambientes urbanos de Santiago. Ambiente y Desarrollo, 19(2):31-38.

Escobedo, F., Nowak, D., Wagner, J., De la Maza, C., Rodríguez, M., Crane, D. y otros (2006). The socioeconomics and management of Santiago de Chile's public urban forests. Urban Forestry \& Urban Greening 4, 105-114.

Figueroa, I. (2009). Conectividad y accesibilidad de los espacios abiertos urbanos en Santiago de Chile. Tesis para optar al Grado de Magister en Asentamientos Humanos y Medio Ambiente. Santiago: Instituto de Estudios Urbanos y Territoriales. Pontificia Universidad Católica de Chile.

Handley, J., Pauleit, S., Slinn, P., Barber, A., Baker, M., Jones, C. y otros (2003). Accessible Natural Green Space Standards in Town and Cities: A Review and Toolkit for their Implementation. Peterborough UK: English Nature Report Number 526. 
Hernández, J. (2007). La situación del arbolado urbano en Santiago. Ambiente Forestal 3, 14-16.

Hough, M. (1998). Naturaleza y Ciudad. Barcelona: Gustavo Gili.

INE. (2005). Ciudades, Pueblos, Aldeas y Caserios. Santiago de Chile: Instituto Nacional de Estadísticas.

Jenerette, G. D., Harlan, L. S., Brazel, A., Jones, N., Larsen, L. \& Stefanov, W. L. (2007). Regional relationships between surface temperature, vegetation and human settlement in a rapidly urbanizing ecosystems. Landscape Ecology 22, 353-365.

Knapp, S., Kühn, I., Mosbrugger, V. \& Klotz, S. (2008). Do protected areas in urban and rural landscapes differ in species diversity? Biodiversity Conservation 17, 1595-1612.

Kühn, I., Brandl, R. \& Klotz, S. (2004). The flora of German cities is naturally species rich. Evolutionary Ecology Research 6, 749-764.

Maas, J., Sonja ME, V. D., Robert A, V. \& Groenewegen, P. P. (2009). Social contacts as a mechanism behind the relation between green space and health. Health and Place 15, 586-595.

Mäkinen, K. \& Tyrväinen, L. (2008). Teenage experience of public green spaces in suburban Helsinki. Unban Forestry \& Urban Greening 7, 277-289.

Ministerio de Vivienda y Urbanismo. (2010). Ordenanza General de Urbanismo y Construcciones. Actualizada al 16 de enero de 2010. Recuperado el 19 de julio de 2010, de sitio web Ministerio de Vivienda y Urbanismo de Chile: http://www.minvu.cl/opensite_20070317162627.aspx

Neel, M. C., McGarigal, K. \& Kushman, S. A. (2004). Behaviour of class-level landscape metric across gradients of class aggregation and area. Landscape Ecology 19, 435-455.

Nilo, C. (2003). Plan Verde: Un instrumento para la gestión y el fomento de áreas verdes en el Gran Santiago. Urbano Año $6 N^{\circ} 8$. Disponible en http://redalyc.uaemex.mx/pdf/198/19800805.pdf.

Pauchard, A., Aguayo, M. \& Alaback, P. (2006). Cuantificando la fragmentación del paisaje: las métricas y sus significados ecológicos. En A. Grez, J. Simonetti, \& R. Bustamante (Eds.). Biodiversidad en ambientes fragmentados de Chile: patrones y procesos a diferentes escalas: 41-67. Santiago: Editorial Universitaria.

Romero, H. \& Vásquez, A. (2005). Evaluación ambiental del proceso de urbanización de las cuencas del piedemonte andino de Santiago de Chile. EURE XXI(94), XXI, 97-118.

Roovers, P.; Hermy, M. \& Gulinck, H. (2002). Visitor profile, perceptions and expectations in forest from gradient of increasing urbanization in central Belgium. Landscape and Urban Planning 59, 129-145.

Sabatini, F., Vásquez, H., Robles, S. \& Rasse, A. (2010). Gentrificación sin expulsión, fuerza de transformación de ciudades latinoamericanas: datos e interpretación para Santiago. En F. Sabatini, R. Salcedo, G. Wormald \& G. Cáceres (Eds.). Tendencias de la Segregación en las Principales Ciudades Chilenas (186). Santiago: Pontificia Universidad Católica de ChileInstituto Nacional de Estadísticas.

Salvador P., P. (2003). La planificación verde en las ciudades. Barcelona: Gustavo Gili.

Saura, S. \& Martínez Millán, J. (2001). Sensitivity of landscape pattern metrics to map spatial extent. Photogrammetric Engineering and Remote Sensing 67, 1027-1036.

Sorensen, M., Berzatti, V., Kerpi, K. \& Williams, J. (1998). Manejo de las Áreas Verdes Urbanas. Washington: Banco Interamericano de Desarrollo. Departamento de Desarrollo Sostenible $\mathrm{N}^{\circ}$ ENV-109.

Sugiyama, T. \& Ward Thompson, C. (2008). Associations between characteristics of neighbourhood open space and older people’s walking. Urban Forestry \& Urban Greening 7, 41-51. 
Sukopp, H. \& Werner, P. (1991). Naturaleza en las ciudades: Desarrollo de flora y fauna en áreas urbanas. Madrid: MOPT Secretaría de Estado para las Políticas del Agua y el Medio Ambiente.

Tahvanainen, L., Tyrväinen, L., Ihalainen, M., Vuorela, N. \& Kolehmainen, O. (2001). Forest management and public perceptions: visual versus verbal information. Landscape and Urban Planning 53, 53-70.

Universidad de Chile (2008). Encuesta de ocupación y desocupación en La Pintana. Recuperado el 19 de julio de 2010, de sitio web Municipalidad de la Pintana: http://www.pintana.cl/images/ stories/doc/encuestajunio2008.pdf

White, J. G., Antos, M. J., Fitzsimons, J. A. \& Palmer, G. C. (2005). Non-uniform bird assemblages in urban environments: the influence of streetscape vegetation. Landscape and Urban Planning $71,123-135$.

\section{Páginas web:}

www.sinim.cl SINIM Sistema Nacional de Indicadores Municipales.

www.observatoriourbano.cl 
Anexo

CUADRO A1 | Resultados de los indicadores aplicados en las comunas del AMS

\begin{tabular}{|c|c|c|c|c|c|c|c|c|}
\hline \multirow{2}{*}{ COMUNAS AMS } & \multirow{2}{*}{$\begin{array}{c}\text { NÚMERO } \\
\text { DE ÁREAS } \\
\text { VERDES } \\
\text { NP }\end{array}$} & \multirow{2}{*}{$\begin{array}{c}\text { SUPERFICIE } \\
\text { DE ÁREAS } \\
\text { VERDES } \\
\text { CA }\end{array}$} & \multirow{2}{*}{$\begin{array}{c}\text { PARTICIPACIÓN } \\
\text { EN LA } \\
\text { SUPERFICIE } \\
\text { TOTAL DE AV } \\
\text { PLAND }\end{array}$} & \multirow{2}{*}{$\begin{array}{l}\text { ÍNDICE DEL } \\
\text { PARCHE } \\
\text { MÁs } \\
\text { GRANDE } \\
\text { LPI }\end{array}$} & \multirow{2}{*}{$\begin{array}{c}\text { ÍNDICE DE } \\
\text { COHESIÓN } \\
\text { IC }\end{array}$} & \multirow{2}{*}{$\begin{array}{c}\text { ÍNDICE } \\
\text { DEL } \\
\text { VECINO } \\
\text { MÁS } \\
\text { CERCANO } \\
\text { NNR } \\
\end{array}$} & \multicolumn{2}{|c|}{$\begin{array}{c}\text { Porcentaje de } \\
\text { POBLACIÓN COMUNAI }\end{array}$} \\
\hline & & & & & & & INDIGENTE & Pobre \\
\hline Cerrillos & 191 & 134,9 & $3,53 \%$ & 39,8 & 99,46 & 0,73 & 2,7 & 8,3 \\
\hline Cerro Navia & 137 & 33 & $0,86 \%$ & 9,4 & 98,5 & 0,77 & 3,5 & 17,5 \\
\hline Conchalí & 306 & 49,9 & $1,31 \%$ & 4,5 & 98,04 & 0,72 & 1,1 & 8,0 \\
\hline EL BOSQUE & 162 & 31 & $0,81 \%$ & 19,4 & 98,25 & 0,76 & 3,7 & 15,8 \\
\hline $\begin{array}{l}\text { Estación } \\
\text { CENTRAL }\end{array}$ & 303 & 82,8 & $2,16 \%$ & 9,5 & 98,66 & 0,69 & 1,3 & 7,3 \\
\hline HUECHURABa & 218 & 84,4 & $2,21 \%$ & 56,9 & 99,4 & 0,45 & 2,8 & 14,5 \\
\hline INDEPENDENCIA & 133 & 17,4 & $0,45 \%$ & 6,1 & 97,88 & 0,6 & 1,3 & 6,0 \\
\hline La Cisterna & 52 & 39,8 & $1,04 \%$ & 59,8 & 99,36 & 0,89 & 0,8 & 8,6 \\
\hline LA FLORIDA & 721 & 119,4 & $3,12 \%$ & 11,1 & 98,14 & 0,7 & 1,6 & 9,6 \\
\hline LA GRANJA & 240 & 81,2 & $2,12 \%$ & 57,2 & 99,23 & 0,82 & 4,6 & 14,2 \\
\hline La Pintana & 244 & 46,1 & $1,20 \%$ & 12,1 & 98,29 & 0,61 & 3,6 & 17,2 \\
\hline LA REINA & 225 & 190,1 & $4,97 \%$ & 33,8 & 99,48 & 0,72 & 0,5 & 7,8 \\
\hline LAS CONDES & 724 & 221,8 & $5,80 \%$ & 24,5 & 98,75 & 0,65 & 0,6 & 2,3 \\
\hline LO BARNECHEA & 335 & 64,3 & $1,68 \%$ & 6,8 & 98,65 & 0,56 & 2,9 & 8,1 \\
\hline Lo EsPEJo & 165 & 30,9 & $0,81 \%$ & 10,7 & 98,57 & 0,79 & 4,8 & 20,1 \\
\hline Lo Prado & 192 & 35,7 & $0,93 \%$ & 9,3 & 98,35 & 0,78 & 3,8 & 11,6 \\
\hline Macul & 225 & 49,4 & $1,29 \%$ & 18,5 & 98,38 & 0,9 & 0,7 & 13,4 \\
\hline MaIPÚ & 1,701 & 238,6 & $6,24 \%$ & 3,5 & 98,07 & 0,68 & 2,1 & 9,1 \\
\hline NUUŃOA & 391 & 141,4 & $3,70 \%$ & 45 & 98,82 & 0,71 & 0,9 & 4,3 \\
\hline $\begin{array}{r}\text { P. AgUiRRe } \\
\text { CERDA }\end{array}$ & 186 & 55,3 & $1,44 \%$ & 20,5 & 98,82 & 0,78 & 1,8 & 6,3 \\
\hline Peñalolén & 602 & 190,1 & $4,97 \%$ & 28,4 & 98,88 & 0,69 & 3,2 & 8,7 \\
\hline Providencia & 279 & 218,3 & $5,71 \%$ & 66,7 & 99,49 & 0,67 & 0,0 & 3,5 \\
\hline Pudahuel & 189 & 56 & $1,46 \%$ & 10,4 & 98,61 & 0,81 & 1,2 & 7,1 \\
\hline Puente Alto & 1.613 & 183,7 & $4,80 \%$ & 5 & 97,69 & 0,57 & 3,0 & 10,6 \\
\hline QUILICURA & 300 & 48,3 & $1,26 \%$ & 5,2 & 98,12 & 0,79 & 0,8 & 6,7 \\
\hline $\begin{array}{c}\text { QUINTA } \\
\text { NORMaL }\end{array}$ & 64 & 25,3 & $0,66 \%$ & 23,6 & 98,92 & 0,86 & 2,8 & 10,8 \\
\hline RECOLETA & 252 & 293,1 & $7,66 \%$ & 77,4 & 99,77 & 0,57 & 1,3 & 12,4 \\
\hline RENCA & 128 & 238,3 & $6,23 \%$ & 84,9 & 99,76 & 0,64 & 2,5 & 19,2 \\
\hline SAN BERNARDO & 333 & 76,6 & $2,00 \%$ & 3 & 98,45 & 0,55 & 5,8 & 20,9 \\
\hline SAN JOAQUín & 157 & 35,6 & $0,93 \%$ & 11,1 & 98,45 & 0,79 & 2,1 & 7,4 \\
\hline SAN MigUEL & 72 & 21,4 & $0,56 \%$ & 14,2 & 98,63 & 0,75 & 1,4 & 2,5 \\
\hline SAN RAMÓN & 135 & 41,9 & $1,09 \%$ & 15,3 & 98,77 & 0,84 & 4,4 & 16,7 \\
\hline SANTIAGO & 265 & 191,2 & $5,00 \%$ & 20,6 & 99,24 & 0,7 & 1,5 & 7,3 \\
\hline VITACURA & 362 & 458,1 & $11,98 \%$ & 22,8 & 99,49 & 0,71 & 3,7 & 4,4 \\
\hline
\end{tabular}

"Se considera pobre si su nivel de ingreso se sitúa por debajo de un nivel mínimo que le permita satisfacer sus necesidades básicas; e indigente, si éste no le permite satisfacer sus necesidades alimentarias". Disponible en:

http://www.mideplan.cl/admin/docdescargas/centrodoc/centrodoc_16.pdf

FUENTE FIGUEROA, 2009 CASEN, 2006 